Research Paper

\title{
Prognostic Significance of Forkhead Box M1 (FOXM1) Expression and Antitumor Effect of FOXM1 Inhibition in Angiosarcoma
}

\author{
Takamichi Ito ${ }^{1,2}$, Kenichi Kohashi ${ }^{1}$, Yuichi Yamada ${ }^{1}$, Takeshi Iwasaki ${ }^{1}$, Akira Maekawa1 ${ }^{1}$ Masaaki Kuda1, \\ Daichi Hoshina ${ }^{3}$, Riichiro Abe ${ }^{3}$, Masutaka Furue ${ }^{2}$ and Yoshinao Oda ${ }^{1 凶}$ \\ 1. Department of Anatomic Pathology, Graduate School of Medical Sciences, Kyushu University, Fukuoka, Japan; \\ 2. Department of Dermatology, Graduate School of Medical Sciences, Kyushu University, Fukuoka, Japan; \\ 3. Department of Dermatology, Hokkaido University Graduate School of Medicine, Sapporo, Japan. \\ $\square$ Corresponding author: Yoshinao Oda, MD, PhD. Address: Department of Anatomic Pathology, Graduate School of Medical Sciences, Kyushu University, \\ 3-1-1, Maidashi, Higashi-ku, Fukuoka 812-8582, Japan. Tel.: (81) 92-642-6061, Fax: (81) 92-642-5968, E-mail: oda@surgpath.med.kyushu-u.ac.jp.
}

(1) Ivyspring International Publisher. Reproduction is permitted for personal, noncommercial use, provided that the article is in whole, unmodified, and properly cited. See http://ivyspring.com/terms for terms and conditions.

Received: 2015.11.17; Accepted: 2016.03.15; Published: 2016.04.27

\begin{abstract}
Background: The prognosis of angiosarcoma is poor and a novel treatment option for the disease is desired. The aim of this study was to investigate the prognostic significance of Forkhead box MI (FOXMI), a transcription factor that regulates cell-cycle progression and various crucial processes in tumor progression, and its potential as a new therapeutic target.

Methods: We investigated 125 angiosarcoma clinical samples (94 primary lesions and 31 metastatic lesions in 94 patients) and a human angiosarcoma cell line (HAMON) using immunohistochemical staining and molecular biological approaches. FOXMI expression in angiosarcoma samples was also compared with that in Kaposi's sarcomas $(n=13)$, epithelioid hemangioendotheliomas $(n=13)$ and benign hemangiomas $(n=10)$.

Results: Patients with FOXMI-overexpressing angiosarcoma had significantly shorter survival (both for disease-specific survival [DSS] and event-free survival [EFS]) than other patients (5-year DSS, 23.5\% vs. $47.1 \%, P=0.013$; and 5 -year $E F S, 5.5 \%$ vs. $28.7 \%, P=0.004$ ). FOXMl overexpression was also an independent prognostic factor for both DSS and EFS in Cox multivariate analyses (hazard ratio [HR] 2.84, 95\% confidence interval $[\mathrm{Cl}] 1.10-5.81, \mathrm{P}=0.039$; and $\mathrm{HR} 4.16,95 \% \mathrm{Cl} 2.03-8.67, \mathrm{P}=0.0001$, respectively). FOXMI inhibition using both small interfering RNA and a specific inhibitor (thiostrepton) suppressed cell proliferation of the angiosarcoma cell line. Furthermore, FOXMI inhibition improved the chemosensitivity to docetaxel in vitro.
\end{abstract}

Conclusions: FOXMI inhibition may be a potential therapeutic option for angiosarcoma.

Key words: Forkhead box M1, angiosarcoma, Kaposi's sarcoma, prognosis, treatment, siRNA, docetaxel, thiostrepton.

\section{Introduction}

Angiosarcoma is a rare, malignant vascular sarcoma accounting for $<2 \%$ of all soft tissue sarcomas, ${ }^{1}$ and is one of the most aggressive malignant tumors. The prognosis of angiosarcoma is dismal with a 5-year survival rate of $10-35 \% .^{2-4}$ The therapy for angiosarcoma requires a multimodal approach, including surgical resection, radiotherapy, and chemotherapy. ${ }^{2,}$ 5-7 Although recent emerging molecular-targeted therapies have yielded some advances in the treatment of angiosarcoma patients, the prognosis is still poor. ${ }^{8-10}$ Therefore, a new treatment option for advanced angiosarcoma is still desired.

Forkhead box M1 (FOXM1) is a member of the FOX family of transcription factors and is widely expressed in embryonic tissues. ${ }^{11-13}$ Controlled 
FOXM1 expression and activity provides a balanced transcriptional program to ensure proper growth and maturation throughout embryonic and fetal development, as well as during adult tissue homeostasis and repair. FOXM1 also regulates crucial processes in both development and tumor progression, including stem cell renewal, cell proliferation, tumor progression, cell migration, tumor metastasis, epithelial-mesenchymal transition, angiogenesis, and chemoresistance. ${ }^{11-13}$ Previous studies have revealed that FOXM1 is overexpressed in various cancers and sarcomas such as breast cancer, lung cancer, pancreatic cancer, glioblastoma, and non-Hodgkin's lymphoma. ${ }^{12-17}$ Overexpression of FOXM1 is a poor prognostic factor in many of these tumors. In contrast, limited information is available on the association of FOXM1 expression in angiosarcoma, and its role in tumorigenesis or tumor progression has not been fully elucidated.

In this study, we first examined FOXM1 overexpression in clinical specimens by immunohistochemistry to investigate the prognostic significance of FOXM1 in angiosarcoma patients. Furthermore, we performed in vitro assays using an angiosarcoma cell line, thereby demonstrating the potential of FOXM1 as a new therapeutic target.

\section{Materials and Methods}

\section{Patients and tissue samples}

This study was conducted in accordance with the principles embodied in the Declaration of Helsinki, and was approved by the Ethics Committee of Kyushu University (No. 27-78).

We examined 125 paraffin-embedded angiosarcoma clinical samples (94 primary lesions and 31 metastatic lesions in 94 patients) registered in the Department of Anatomic Pathology, Graduate School of Medical Sciences, Kyushu University, Japan, between 1971 and 2014. We also investigated FOXM1 expression in 13 samples of epithelioid hemangioendothelioma, 13 samples of Kaposi's sarcoma and 10 samples of benign hemangioma for comparison. Clinical and demographic data were retrieved from the patient files. Patients with epithelioid hemangioendothelioma, Kaposi's sarcoma and benign hemangioma were excluded from all of the survival analyses.

Follow-up data were available for 59 angiosarcoma patients; at the last follow up, 29 patients were alive, 17 of whom had not experienced metastasis, 30 patients had died of angiosarcoma, and none had died of other causes. Patients included in the survival analyses were relatively recent cases and primarily underwent radiation therapy (45-85 Gy), followed by taxane-based chemotherapy or intravascular interleukin-2 injection without the radical surgical treatment. Total resection of the primary tumor was performed in only six patients; all six of their primary angiosarcomas were successfully excised with negative surgical margins. Disease-specific survival (DSS) and event-free survival (EFS) were calculated from the date of the first histopathological examination to the date of death due to angiosarcoma, or the date of recurrence or metastasis. Data of patients without death or recurrence were censored on the date of the last follow-up before March 31, 2015. The median follow-up periods were 18 months (mean, 27.2 months) for DSS and 14 months (mean, 22.1 months) for EFS. For surviving patients, the median follow-up period was 28 months (mean, 35.6 months).

\section{Immunohistochemical analysis}

All samples were fixed with $10 \%$ buffered formalin. The archival paraffin-embedded tissue blocks were cut into $3-\mu \mathrm{m}$-thick tissue sections. The sections were deparaffinized with xylene for $10 \mathrm{~min}$ and rehydrated through a graded ethanol series. Antigen retrieval was performed using Heat Processor Solution pH6 (Nichirei Biosciences, Tokyo, Japan) at $125^{\circ} \mathrm{C}$ for $10 \mathrm{~min}$ by a pressure cooker, and non-specific binding was blocked using the supernatant of $1 \%$ skimmed milk. The sections were then incubated with a rabbit antibody against human FOXM1 (1:1000, Santa Cruz Biotechnology, Santa Cruz, CA) and Ki-67 (1:100, DAKO, Glostrup, Denmark) at $4^{\circ} \mathrm{C}$ overnight, followed by incubation with the secondary antibody, N-Histofine Simple Stain AP MULTI (Nichirei Biosciences). Immunodetection was conducted with a standard streptavidin-biotin amplification method and 3-amino-9-ethylcarbazole as a chromogen, followed by light counterstaining with hematoxylin. Sections stained without primary antibody served as a negative control.

After immunohistochemical staining, three pathologists (T.I., Y.Y., and K.K.) who were blind to the patient details, evaluated the stained sections. The immunoreactivity of FOXM1 was defined as cells showing nuclear staining with or without cytoplasmic staining patterns. Tumors showing strong staining intensity in $>10 \%$ of the tumor cells were determined to show positive immunoreactivity based on a previously reported method. ${ }^{18,19}$ The FOXM1 staining intensity was judged in comparison to FOXM1-positive keratinocytes in the epidermis or non-neoplastic cells expressing FOXM1 as an internal control. The Ki-67 labeling index was calculated as described previously. ${ }^{20}$ 


\section{Cell culture of angiosarcoma cells}

Human angiosarcoma HAMON cells ${ }^{21}$ were cultured in Endothelial Cell Growth Medium (EGM-2 BulletKit; Lonza, Basel, Switzerland). HAMON is Cells were grown in culture dishes at $37^{\circ} \mathrm{C}, 5 \% \mathrm{CO}_{2}$. The culture medium was replaced every 2 days. At near confluence (70-90\%), the cells were disaggregated with $0.25 \mathrm{mg} / \mathrm{ml}$ trypsin/0.01\% ethylenediamine tetraacetic acid and subcultured.

\section{Drugs}

Docetaxel was purchased from Wako Pure Chemicals (Osaka, Japan), and thiostrepton, a FOXM1 inhibitor, was obtained from Millipore (Bedford, MA, USA). Both drugs were dissolved in dimethyl sulfoxide (DMSO; Sigma-Aldrich, St. Louis, MO, USA) and were used at the indicated concentrations.

\section{Small interfering RNA (siRNA) transfection}

The angiosarcoma cells were transfected with On-Target plus Smart Pool siRNAs FOXM1 and On-Target plus Smart Pool siRNAs siControl (GE Healthcare, Buckinghamshire, UK.) using Lipofectamine RNA imax (Invitrogen, Carlsbad, CA, USA) according to the manufacturer's protocols. Angiosarcoma cells were seeded in 6-well plates at a concentration of $1 \times 10^{5}$ cells per well in antibiotics-free medium and transfected with $10 \mathrm{pmol}$ siRNAs. The transfection of the siRNA for FOXM1 (siFOXM1) was confirmed by quantitative reverse transcription-polymerase chain reaction (qRT-PCR) and immunoblotting.

\section{Real-time PCR assay}

We performed qRT-PCR for FOXM1, which was analyzed using TaqMan assay reagents (FOXM1 Hs00170471_m1.; GAPDH Hs99999905_m1.; Applied Biosystems, Foster City, CA, USA) on an ABI Prism 7700 Sequence Detection system (Applied Biosystems). The PCR was carried out according to the manufacturer's protocol. The FOXM1 expression levels were standardized based on expression of the housekeeping gene GAPDH. All of the reactions for each sample were performed at least in triplicate. The data were averaged from the values obtained in each reaction.

\section{Western blotting}

Angiosarcoma cells were incubated with lysis buffer (Complete Lysis-M, Roche Applied Science, Indianapolis, IN, USA). The lysate protein concentration was measured using a BCA Protein Assay kit (Pierce, Rockford, IL, USA). Equal amounts of protein $(20 \mu \mathrm{g})$ were dissolved in NuPage LDS Sample Buffer (Invitrogen) and 10\% NuPage Sample
Reducing Agent (Invitrogen). Lysates were boiled at $98^{\circ} \mathrm{C}$ for 2 minutes and loaded and run on $4-12 \%$ NuPage Bis-Tris Gels (Invitrogen) at $200 \mathrm{~V}$ for 40 minutes. The proteins were transferred onto polyvinylidene fluoride membranes (Invitrogen) and blocked in $2 \%$ bovine serum albumin in $0.1 \%$ Tween-20 (Sigma-Aldrich) and Tris-buffered saline. The membranes were probed with anti-FOXM1 antibody (1:100, Santa Cruz) overnight at $4^{\circ} \mathrm{C}$. The secondary antibody used was anti-rabbit horseradish peroxidase-conjugated IgG antibody. Anti-human actin mouse monoclonal antibody (1:4000, Millipore) was used as a loading control. Protein bands were detected using the ImageQuant LAS 4000 system (GE Healthcare).

\section{Cell viability}

Cell viability was assessed using Cell Counting Kit 8 (CCK-8, Dojindo Molecular Technologies, Rockville, MD, USA) according to the manufacturer's instructions. The absorbance at $450 \mathrm{~nm}$ was spectrophotometrically measured with a microplate reader (Model 680, Bio-Rad Laboratories, Hercules, CA, USA) at $450 \mathrm{~nm}$.

\section{Proliferation assay and chemosensitivity of the transfected cell lines}

Twenty-four hours after siRNA transfection, the transfected cells were seeded at a density of 5000 cells per well in 96-well plates. For the proliferation assay, the number of viable cells in each well was measured at 36,60 , and 84 hours after transfection using CCK- 8 (Dojindo).

\section{Proliferation assay and chemosensitivity of FOXM1-inhibitor treatment}

The angiosarcoma cells were seeded on 96-well plates at a concentration of 3000 cells per well in serum-containing growth medium. After a 12-hour incubation, the cells were treated with carrier alone $(0.01 \%$ DMSO) as a non-treated control or with various concentrations of thiostrepton for another 72 hours. The resulting data are reported as the percentage of cell viability in comparison to that of the respective non-treated control group $(100 \%)$.

For the chemosensitivity assay, we added docetaxel $(0.25 \mu \mathrm{M}$ or $0.5 \mu \mathrm{M})$ with or without thiostrepton $(0.5 \mu \mathrm{M})$ and measured the number of viable cells after another 72 hours using CCK-8 (Dojindo). The concentrations of these agents were determined based on a pilot assay. Assays were conducted in triplicate and were repeated at least three times in separate experiments.

\section{Statistical analysis}

All statistical analyses were performed using the 
JMP Pro statistical software package (version 11.0, SAS Institute, Cary, NC, USA) and the GraphPad Prism statistical software package (version 6, GraphPad Software, San Diego, CA, USA).

The associations between FOXM1 expression and other clinicopathological parameters, including age, gender, primary tumor site, definite cause, histopathological type, and TNM stage, were assessed using the $\chi^{2}$ or Fisher exact test as appropriate. We used the Kaplan-Meier method to evaluate DSS and EFS, and compared survival curves using the log-rank test. The influence of FOXM1 expression on survival was also analyzed using multivariate Cox proportional hazards regression models. The risk factors (covariates) were considered to be FOXM1 expression, age, gender, primary tumor site, histopathological subtype, and TNM stage. Cell viabilities were analyzed by one-way analysis of variance (ANOVA) followed by Dunn's multiple comparison post-hoc test or the Mann-Whitney U-test as appropriate. A P-value of less than 0.05 was considered to indicate statistical significance.

\section{Results}

\section{Patient data}

We investigated 125 angiosarcoma clinical samples (94 primary lesions and 31 metastatic lesions in 94 patients) and 13 Kaposi's sarcoma clinical samples (13 patients); 63 patients $(58.9 \%)$ were male and $44(41.4 \%)$ were female, with a mean age of 66.3 years. Primary tumor sites were the skin in 76 tumors $(71.0 \%)$ and other sites in 31 tumors (29.0\%). Radiation-induced angiosarcoma was noted in one patient $(0.9 \%)$ and lymphedema-associated angiosarcoma (Stewart-Treves syndrome) was confirmed in three patients $(2.8 \%)$. Histopathologically epithelioid features were observed in 17 patients (15.9\%). We did not distinguish two phenotypes of angiosarcoma (lymphangiosarcoma and hemangiosarcoma) because of the insufficient assessment from small clinical samples.

\section{Immunohistochemical results}

Representative histopathological images of benign hemangioma and angiosarcoma are shown in Figure 1. Benign hemangiomas and epithelioid hemangioendotheliomas rarely expressed FOXM1, whereas it was frequently expressed in angiosarcomas and Kaposi's sarcomas. Statistically significant differences in FOXM1 expression were observed between benign hemangioma and primary angiosarcoma, metastatic angiosarcoma, and Kaposi's sarcoma, respectively (one-way ANOVA followed by Dunn's multiple comparison post-hoc test; $\mathrm{P}<0.001$, $\mathrm{P}<0.001$, and $\mathrm{P}<0.01$, respectively). Epithelioid hemangioendothelioma less frequently expressed FOXM1 than primary angiosarcoma and metastatic angiosarcoma ( $\mathrm{P}<0.001, \mathrm{P}<0.001$, respectively). FOXM1 expression was observed significantly more frequently in metastatic angiosarcomas compared with the primary tumors $(\mathrm{P}<0.05)$.

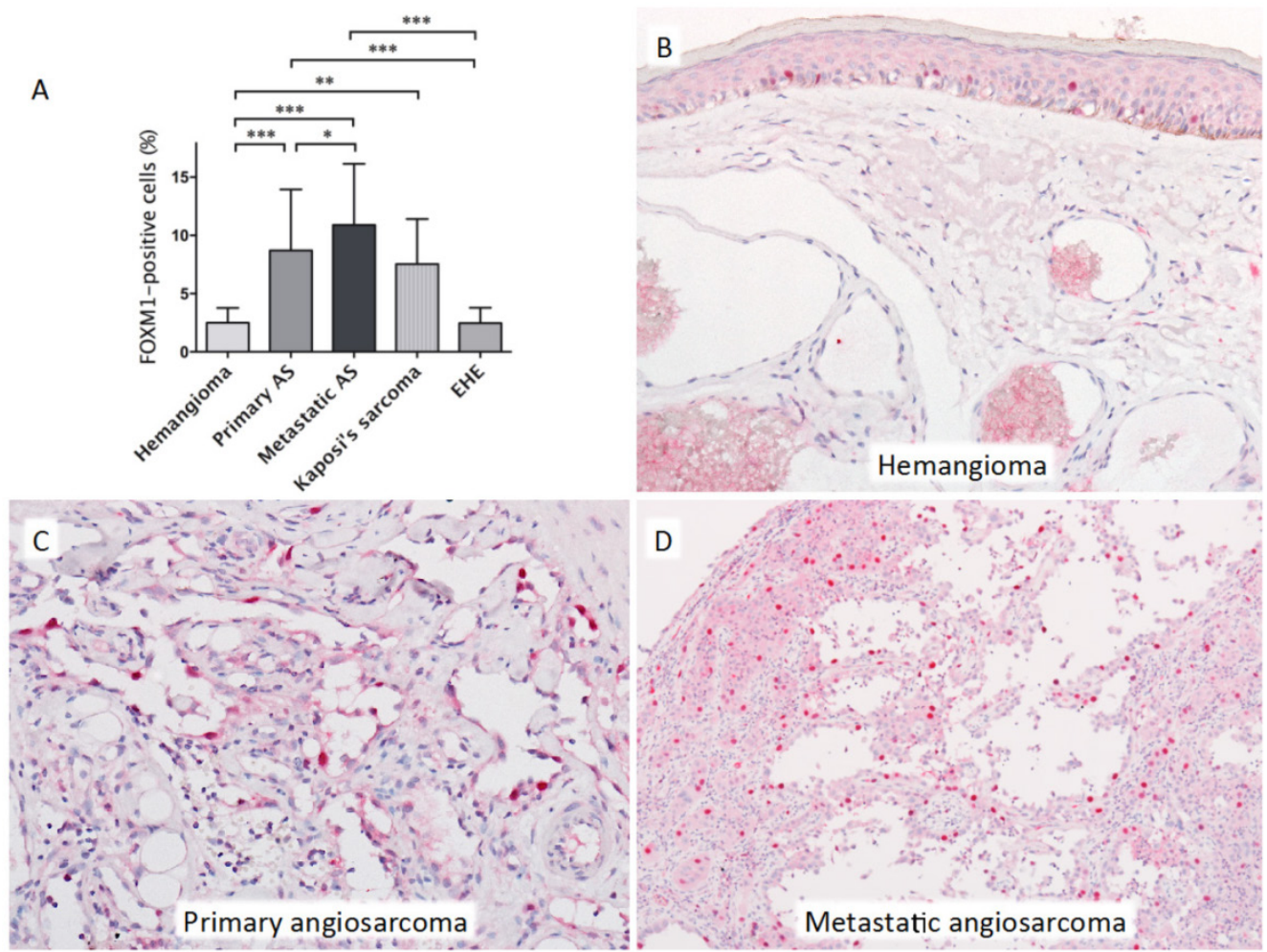

Figure 1. A: Statistically significant differences in FOXM1 expression were observed between samples of benign hemangioma and primary angiosarcoma (AS), metastatic AS, and Kaposi's sarcoma, respectively. Epithelioid hemangioendothelioma (EHE) less frequently expressed FOXM1 compared with primary AS and metastatic AS. There was also a significant difference between primary and metastatic AS (one-way ANOVA followed by Dunn's multiple comparison post-hoc test; $* \mathrm{P}<0.05$, $* * \mathrm{P}<0.01$ and $* * * \mathrm{P}<0.0001)$. Error bars: standard deviation (SD). B: A representative image of a benign hemangioma of the skin showing negative FOXMI expression. Note the strong and frequent nuclear expression of FOXM1 in the epidermal keratinocytes. C: Primary angiosarcoma showing strong FOXM1 expression. D: Metastatic angiosarcoma showing strong FOXMI expression. 

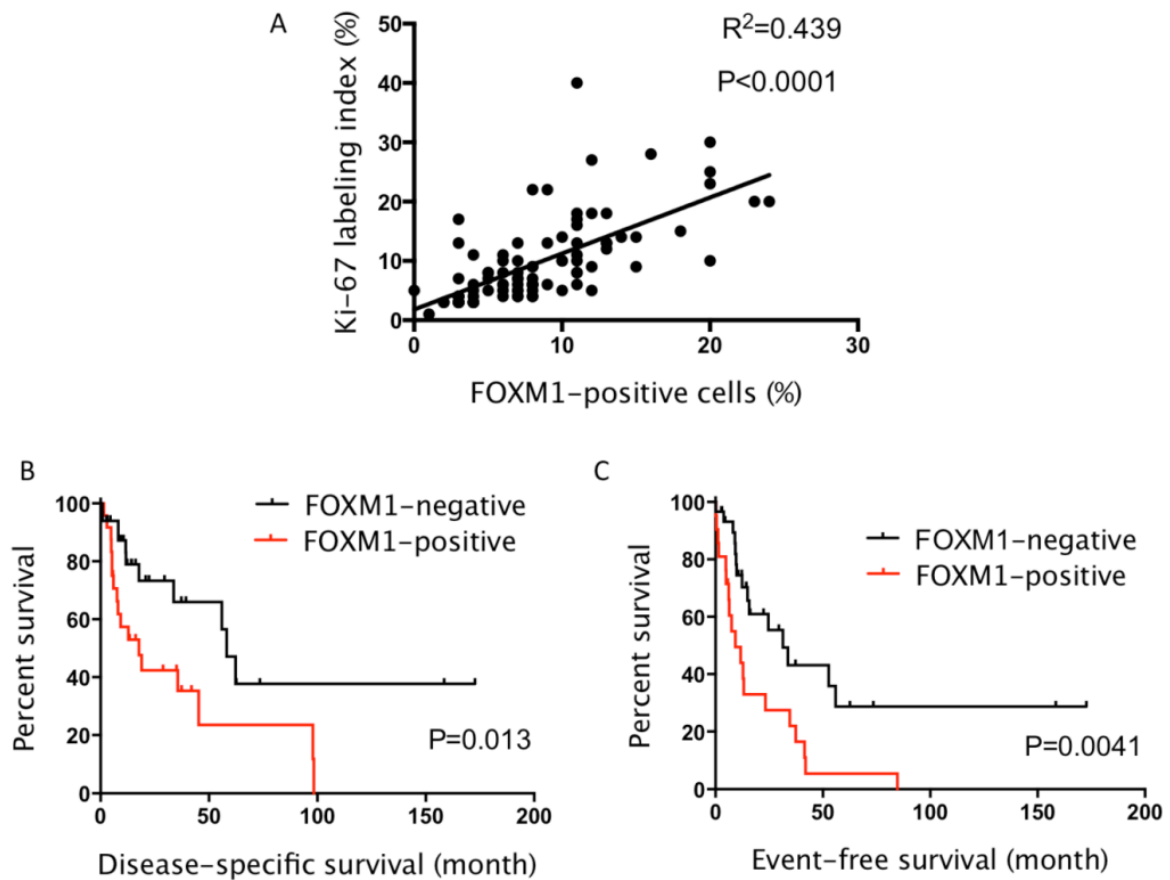

Figure 2. A: A significant correlation between FOXMI and Ki-67 expression was observed. B: The Kaplan-Meier curves of angiosarcoma patients. Patients with FOXM1-high angiosarcoma had significantly shorter disease-specific survival than those without high expression (5-year survival, 23.5\% vs. 47.1\%; P = 0.013). C: Patients with FOXM1-high angiosarcoma had significantly shorter event-free survival than those without high expression (5-year survival, $5.5 \%$ vs. $28.7 \%$; $P=0.004)$.

Since FOXM1 is reportedly associated with cell-cycle progression, we next examined the relationship between FOXM1 and Ki-67 expression, a cell-proliferation marker. A significant correlation was noted between the two markers $\left(\mathrm{R}^{2}=0.439, \mathrm{P}<\right.$ 0.0001; Fig. 2A).

\section{Clinicopathological factors associated with FOXM1 expression in primary angiosarcoma}

Table 1 shows the associations of clinicopathological factors with immunohistochemical FOXM1 expression. Among these factors, advanced TNM stage was significantly correlated with high FOXM1 expression $(\mathrm{P}=0.041)$.

\section{Prognostic impact of FOXMI expression}

Patients with angiosarcoma and high FOXM1 expression had a significantly shorter DSS (5-year survival, $23.5 \%$ vs. $47.1 \%$; $\mathrm{P}=0.013$; Fig. $2 \mathrm{~B}$ ) and EFS (5-year survival, $5.5 \%$ vs. $28.7 \%$; $\mathrm{P}=0.004$; Fig. $2 \mathrm{C}$ ) than those without high expression. We next examined whether FOXM1 affects DSS and EFS in the subgroups of non-advanced angiosarcoma (TNM stage I or II) and advanced angiosarcoma (TNM stage III or IV) patients. Among the patients with non-advanced anigosarcoma, FOXM1 positivity was significantly correlated to the shorter DSS and EFS $(\mathrm{P}=0.049$ and $\mathrm{P}=0.014$, respectively; Supplemental Fig. $\mathrm{S1})$. As for the patients with advanced angiosacoma, the difference of DSS and EFS between FOXM1-positive and FOXM1-negative patients did not reach the statistical significance $(\mathrm{P}=0.709$ and $\mathrm{P}=0.546$, respectively).

Table 1: Factors associated with FOXM1 expression.

\begin{tabular}{|c|c|c|c|}
\hline \multirow[b]{2}{*}{ Parameter } & \multicolumn{3}{|c|}{ FOXM1 expression } \\
\hline & $\leq 10 \%$ & $>10 \%$ & P-value \\
\hline \multicolumn{4}{|l|}{ Age (years) } \\
\hline$<70$ & $31(33.0 \%)$ & $10(10.6 \%)$ & $0.37 \mathrm{a}$ \\
\hline$\geq 70$ & $35(37.2 \%)$ & $18(19.1 \%)$ & \\
\hline \multicolumn{4}{|l|}{ Gender } \\
\hline Male & $35(37.2 \%)$ & $16(17.0 \%)$ & $0.82^{a}$ \\
\hline Female & $31(33.0 \%)$ & $12(12.8 \%)$ & \\
\hline \multicolumn{4}{|l|}{ Primary site } \\
\hline Skin & $44(46.8 \%)$ & $23(24.5 \%)$ & $0.15^{a}$ \\
\hline Non-skin & $22(23.4 \%)$ & $5(5.3 \%)$ & \\
\hline \multicolumn{4}{|l|}{ Definite cause } \\
\hline None & $64(68.1 \%)$ & $26(27.7 \%)$ & $0.30^{b}$ \\
\hline Radiation-induced & $0(0 \%)$ & $1(1.1 \%)$ & \\
\hline Lymphedema -associated & $2(2.1 \%)$ & $1(1.1 \%)$ & \\
\hline \multicolumn{4}{|l|}{ Histopathological type } \\
\hline Non-epithelioid & $56(59.6 \%)$ & $21(22.3 \%)$ & $0.26^{a}$ \\
\hline Epithelioid & $10(10.6 \%)$ & $7(7.4 \%)$ & \\
\hline \multicolumn{4}{|l|}{ TNM stage } \\
\hline I or II & $60(63.8 \%)$ & $21(22.4 \%)$ & $0.041^{\mathrm{a}}$ \\
\hline III or IV & $6(6.4 \%)$ & $7(7.4 \%)$ & \\
\hline Total & $66(70.2 \%)$ & $28(29.8 \%)$ & \\
\hline
\end{tabular}

a Fisher exact test

${ }^{\mathrm{b}} \mathrm{X}^{2}$ test

Significant value is shown in bold

The results of the Cox multivariate analyses for DSS and EFS in angiosarcoma patients are shown in Tables 2 and 3, respectively. In addition to the FOXM1 expression, we chose four variables (age, gender, 
primary tumor site and TNM stage) that are clinically important and reported to be possible prognostic variables. $^{2-6}$ We excluded the histopathological subtype and histopathological degree of differentiation, which are also potential prognostic variables, from the multivariate analyses because most of the paraffin sections were too small in size (punch biopsy specimens) to be fully assessed histopathologically. The Ki67 labeling index was also excluded from the model because of the strong correlation with the FOXM1 expression. Among the variables included in the multivariate analyses, the independent prognostic factors for DSS were patient age $(P=0.003)$, non-skin primary lesion $(P=0.002)$, and FOXM1 overexpression $(\mathrm{P}=0.039)$.

With regard to EFS, the independent prognostic factors were male gender $(P=0.003)$, non-skin primary lesion $(\mathrm{P}<0.0001)$, advanced TNM stage $(\mathrm{P}<$ $0.0001)$, and FOXM1 overexpression $(P=0.0001)$, as shown in Table 3.

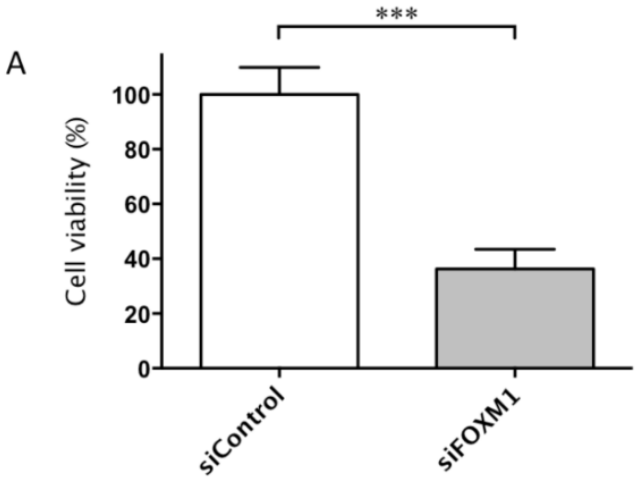

C

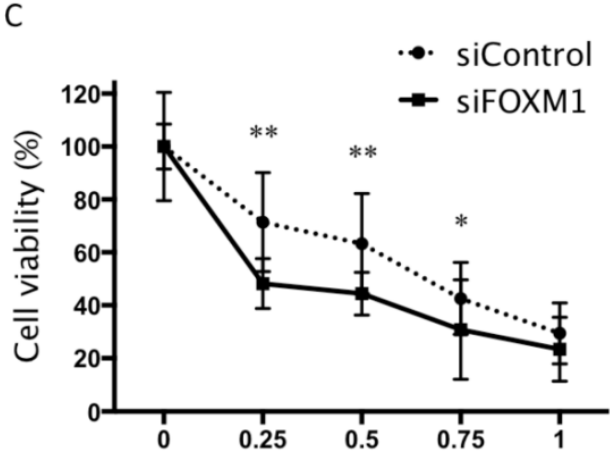

Concentration of docetaxel $(\mu \mathrm{M})$

\section{Antiproliferative effects of siFOXMI and a FOXM1 inhibitor in vitro}

We next examined the influence of FOXM1 on angiosarcoma cells in vitro using transfection siFOXM1 and a FOXM1 inhibitor, thiostrepton, which were confirmed by qRT-PCR and western blotting, respectively (Supplemental Fig. S2).

siFOXM1-transfected HAMON angiosarcoma cells showed reduced cell viability compared to control siRNA-transfected cells $(\mathrm{P}<0.01$; Fig. 3A). Likewise, thiostrepton reduced the cell viability of the angiosarcoma cell line in a dose-dependent manner (Fig. 3B).

Table 2: Factors associated with disease-specific survival (DSS) in the Cox multivariate analyses.

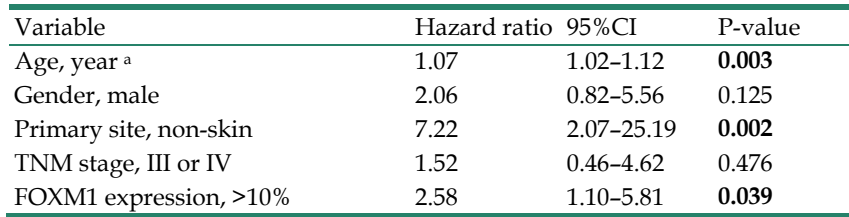

a Expressed as a continuous variable.

Significant values are shown in bold.

B

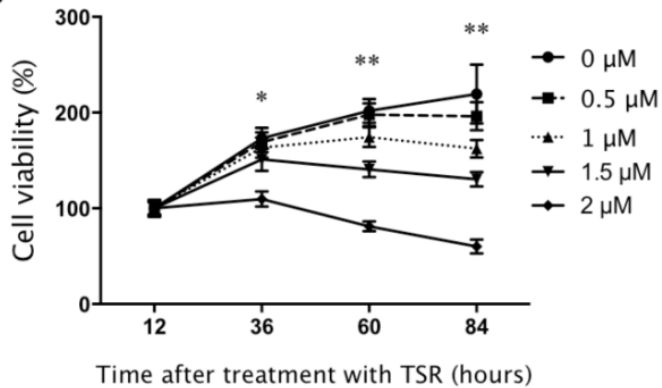

D

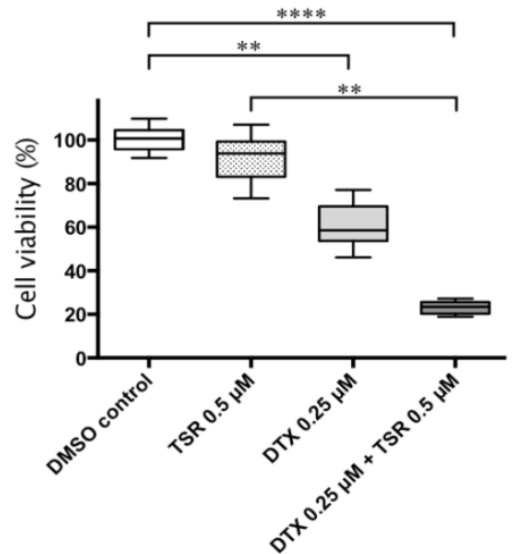

Figure 3. A: siFOXM1-transfected cells showed reduced cell viability than control siRNA-transfected cells (Mann-Whitney U-test; $* * * P<0.001)$. B: Thiostrepton (TSR) reduced cell viability in a dose- dependent manner. We compared the cell viabilities of each group treated with various TRS concentrations at 36,60 and $94 \mathrm{~h}$ (one-way ANOVA followed by Dunn's multiple comparison post-hoc test; *P $<0.05$ and $* * \mathrm{P}<0.01$ ). C: siFOXM1-transfected cells showed suppressed cell viability in response to lower concentrations of docetaxel. We compared the cell viabilities of siFOXMI to those of siControl at the docetaxel concentrations of $0.25,0.5,0.75$ and $1.0 \mu M$ (Mann-Whitney U-test; *P $<0.05$ and **P $<0.01)$. D: Cells treated with both thiostrepton and docetaxel (DTX) showed reduced viability than those treated with DMSO or TSR alone at 72 hours after the treatment. The cells treated with both thiostrepton and docetaxel also showed significantly lower cell viability compared with cells treated with thiostrepton alone. There was no significant difference in cell viability between DMSO-treated cells and thiostrepton-treated cells (one-way ANOVA followed by Dunn's multiple comparison post-hoc test; $* * \mathrm{P}<0.01$ and $* * * * \mathrm{P}<0.0001)$. Error bars: SD. 
Table 3: Factors associated with event-free survival (EFS) in the Cox multivariate analyses.

\begin{tabular}{llll}
\hline Variable & Hazard ratio & $95 \% \mathrm{CI}$ & P-value \\
\hline Age, year a & 1.03 & $0.99-1.06$ & 0.098 \\
Gender, male & 3.04 & $1.47-6.56$ & $\mathbf{0 . 0 0 3}$ \\
Primary site, non-skin & 15.57 & $4.69-52.74$ & $<\mathbf{0 . 0 0 0 1}$ \\
TNM stage, III or IV & 34.63 & $10.29-121.70$ & $<\mathbf{0 . 0 0 0 1}$ \\
FOXM1 expression, $>10 \%$ & 4.16 & $2.03-8.67$ & $\mathbf{0 . 0 0 0 1}$
\end{tabular}

a Expressed as a continuous variable.

Significant values are shown in bold.

\section{Improved chemosensitivity by siFOXMI and FOXM1-inhibitor}

We next tested whether FOXM1 inhibition affects the chemoresistance in angiosarcoma cells. Both siFOXM1-transfected cells and thiostreptontreated cells showed suppressed cell proliferation in significantly lower concentrations of docetaxel, compared to their controls $(\mathrm{P}<0.01$, respectively; Fig. 3C, 3D). Of note, the cells treated with both thiostrepton and docetaxel showed the lowest viability among groups treated with DMSO, thiostrepton alone, or docetaxel alone at 72 hours after the treatment. The cells treated with both thiostrepton and docetaxel demonstrated significantly lower cell viability compared to those treated with DMSO or thiostrepton alone $(\mathrm{P}<0.01$ and $\mathrm{P}<0.0001$, respectively). The difference in cell viability between cells treated with docetacel alone and docetacel with thiostrepton did not reach statistical significance. There was also no significant difference in cell viability between DMSO-treated cells and thiostrepton-treated cells (Fig. 3D).

\section{Discussion}

The present study revealed several important findings. First, FOXM1 overexpression detected by immunohistochemistry was significantly correlated with a poor outcome for angiosarcoma patients. Patients with FOXM1-high angiosarcoma had a significantly shorter survival period with respect to both DSS and EFS than those with FOXM1-low angiosarcoma. High FOXM1 expression was also an independent adverse prognostic factor of DSS and EFS in Cox multivariate analyses. In the subgroup analyses on patients with non-advanced angiosarcoma, FOXM1 positivity was significantly correlated to the shorter DSS and EFS. These results are in good accord with previous studies on other malignant tumors that demonstrated a close association between FOXM1 expression and dismal prognosis, and indicate the prognostic significance of FOXM1 in angiosarcoma.

Another interesting finding is that FOXM1 was more frequently expressed in angiosarcomas than in benign hemangiomas, and was rarely expressed in hemangiomas. Furthermore, metastatic angiosarcomas showed significantly higher levels of FOXM1 than primary angiosarcomas. On the basis of these results, we speculate that FOXM1 might play important roles in tumorigenesis and tumor progression in angiosarcoma, suggesting its potential as a possible therapeutic target. The significant immunohistochemical correlation between Ki-67 and FOXM1 expression provides further support for the involvement of FOXM1 in tumor progression. FOXM1 expression was also frequently observed in the Kaposi sarcomas in this study. Recent studies suggest that human herpesvirus 8 promotes the tumorigenesis of Kaposi sarcoma by modulating the Hippo pathway. ${ }^{22}$ FOXM1 has been shown to be a target of Yes-associated protein (YAP), a downstream effector of the Hippo pathway, in some sacromas, ${ }^{23}$ and deregulation of the Hippo pathway has been shown to promote FOXM1 expression and tumorigenesis. ${ }^{23}$ These findings may suggest a correlation between FOXM1 and the Hippo pathway in the tumorigenesis of vascular sarcomas.

Our clinicopathological findings, which suggest the involvement of FOXM1 in tumor proliferation and progression in angiosarcoma, were further supported by our in vitro experiments. FOXM1 has been shown to be a key player in cell-cycle progression in many cancer cell lines, ${ }^{12-17}$ and our FOXM1-knockdown angiosarcoma cells (transfected with FOXM1 siRNA) showed suppressed cell proliferation relative to the control siRNA-transfected cells. Similar antitumor effects were observed in the cells treated with the FOXM1 inhibitor thiostrepton, which reduced cell viability in a dose-dependent manner. The exact mechanisms by which thiostrepton affects the transcriptional activity and the expression of FOXM1 are still unclear, but it has been postulated that thiostrepton stabilizes a negative regulator of FOXM1 that binds to FOXM1 or acts otherwise to inhibit the transcriptional activity of FOXM1 on its target promoters, including the FOXM1 promoter. ${ }^{24,25}$

Moreover, the FOXM1-inhibitory condition improved the chemosensitivity of angiosarcoma cells to docetaxel. The first-line chemotherapy for advanced angiosarcoma is generally based on taxanes. ${ }^{5-7}$ Angiosarcoma most commonly arises on the skin of the head and neck regions, and previous studies suggest that taxanes are particularly effective for head and neck angiosarcoma compared with angiosarcoma of other regions. Docetaxel is a typical taxane and is widely used for the treatment of angiosarcoma; however, a reduced dosage of docetaxel would be favorable to minimize its adverse effects. Our present in vitro assay demonstrated that 
FOXM1 interruption by siRNA led to a reduction in cell proliferation at lower concentrations of docetaxel. Similar results have been reported in gastric cancer. ${ }^{26}$ The results of that study, it is suggested that FOXM1 directly targets and up-regulates a microtubule-destabilizing protein Stathmin, and then prevents the tubulin polymerization, eventually mediating the resistance to docetaxel-induced apoptosis, and that the FOXM1-induced resistance to docetaxel can be reversed by the FOXM1 inhibition using thiostrepton. ${ }^{26}$ Improved chemosensitivity was also achieved by thiostrepton treatment in our study, and we speculated that similar mechanisms might be at work in the synergistic effects of thiostrepton and docetaxel in angiosarcomas.

Our results from clinical samples and in vitro assays of angiosarcoma are in good agreement with the reported mechanisms of FOXM1 in other malignancies. Ideally, therapeutic intervention should be minimally toxic to normal tissues, and a FOXM1 inhibitor may be suitable from this aspect, because normal adult tissue express low levels of FOXM1. The single use of thiostrepton or a combination of conventional chemotherapy could be another therapeutic option for angiosarcoma showing FOXM1 overexpression.

In addition to the inherent potential biases of a retrospective collection of the patient data, one major limitation of the present study is the relatively small number of patients with available follow-up data. Some of the patients had an extremely short DSS and EFS, and the results of the multivariate analyses should be interpreted with caution.

In summary, we have shown that FOXM1 inhibition is a candidate treatment for angiosarcoma based on the results of our clinicopathological assessment and in vitro study. FOXM1 may be involved in angiosarcoma progression in various ways, and further in vivo and in vitro investigations are warranted to evaluate the efficacy of FOXM1 inhibition alone or in combination with other agents.

\section{Supplementary Material}

Supplementary figures.

http://www.jcancer.org/v07p0823s1.pdf

\section{Abbreviations}

CI, confidence interval; DSS, Disease-specific survival; EFS, event-free survival; FOXM1, Forkhead box M1; HR, hazard ratio.

\section{Acknowledgements}

The authors thank all of the patients, and the members of our laboratory. This work was partly supported by JSPS KAKEN Grant Number 25293088.

\section{Conflict of Interest}

The authors have no conflicts of interest to declare.

\section{References}

1. Albores-Saavedra J, Schwartz AM, Henson DE, et al. Cutaneous angiosarcoma. Analysis of 434 cases from the Surveillance, Epidemiology, and End Results Program, 1973-2007. Ann Diagn Pathol. 2011;15:93-97.

2. Perez MC, Padhya TA, Messina JL, et al. Cutaneous angiosarcoma: a single-institution experience. Ann Surg Oncol. 2013;20:3391-3397.

3. Fayette J, Martin E, Piperno-Neumann S, et al. Angiosarcomas, a heterogeneous group of sarcomas with specific behavior depending on primary site: a retrospective study of 161 cases. Ann Oncol. 2007;18:2030-2036.

4. Fury MG, Antonescu CR, Van Zee KJ, et al. A 14-year retrospective review of angiosarcoma: clinical characteristics, prognostic factors, and treatment outcomes with surgery and chemotherapy. Cancer J. 2005;11:241-247. Erratum in: Cancer J. 2005;11:354

5. Abraham JA, Hornicek FJ, Kaufman AM, et al. Treatment and outcome of 82 patients with angiosarcoma. Ann Surg Oncol. 2007;14:1953-6719.

6. Pawlik TM, Paulino AF, McGinn CJ, et al. Cutaneous angiosarcoma of the scalp: a multidisciplinary approach. Cancer. 2003;98:1716-1726.

7. Fujisawa $\mathrm{Y}$, Yoshino $\mathrm{K}$, Kadono $\mathrm{T}$, et al. Chemoradiotherapy with taxane is superior to conventional surgery and radiotherapy in the management of cutaneous angiosarcoma: a multicentre, retrospective study. Br J Dermatol. 2014;171:1493-1500.

8. Ray-Coquard I, Italiano A, Bompas E, et al; French Sarcoma Group (GSF/GETO). Sorafenib for patients with advanced angiosarcoma: a phase II trial from the French Sarcoma Group (GSF/GETO). Oncologist. 2012;17:260-266.

9. Sleijfer S. Phase II studies in soft tissue sarcoma: time for reappraisal. Oncologist. 2012;17:154-156.

10. Agulnik M, Yarber JL, Okuno SH, et al. An open-label, multicenter, phase II study of bevacizumab for the treatment of angiosarcoma and epithelioid hemangioendotheliomas. Ann Oncol. 2013;24:257-263.

11. Bella L, Zona S, Nestal de Moraes G, et al. FOXM1: A key oncofoetal transcription factor in health and disease. Semin Cancer Biol. 2014;29:32-39.

12. Halasi M, Gartel AL. Targeting FOXM1 in cancer. Biochem Pharmacol 2013;85:644-652.

13. Katoh M, Igarashi M, Fukuda $\mathrm{H}$, et al. Cancer genetics and genomics of human FOX family genes. Cancer Lett. 2013;328:198-206.

14. Pilarsky C, Wenzig $M$, Specht $\mathrm{T}$, et al. Identification and validation of commonly overexpressed genes in solid tumors by comparison of microarray data. Neoplasia 2004;6:744-750.

15. Romagnoli S, Fasoli E, Vaira V, et al. Identification of potential therapeutic targets in malignant mesothelioma using cell-cycle gene expression analysis. Am J Pathol 2009;174:762-770.

16. $\mathrm{Yu} \mathrm{J}$, Deshmukh H, Payton JE, et al. Array-based comparative genomic hybridization identifies CDK4 and FOXM1 alterations as independent predictors of survival in malignant peripheral nerve sheath tumor. Clin Cancer Res. 2011;17:1924-1934.

17. Nakamura S, Hirano I, Okinaka K, et al. The FOXM1 transcriptional factor promotes the proliferation of leukemia cells through modulation of cell cycle progression in acute myeloid leukemia. Carcinogenesis 2010;31:2012-2021.

18. Yang DK, Son CH, Lee SK, et al. Forkhead box M1 expression in pulmonary squamous cell carcinoma: correlation with clinicopathologic features and its prognostic significance. Hum Pathol. 2009;40:464-470.

19. Ha SY, Lee CH, Chang HK, et al. Differential expression of forkhead box M1 and its downstream cyclin-dependent kinase inhibitors p27(kip1) and p21(waf1/cip1) in the diagnosis of pulmonary neuroendocrine tumours. Histopathology 2012;60:731-739.

20. Kobayashi C, Oda Y, Takahira $\mathrm{T}$, et al. Chromosomal aberrations and microsatellite instability of malig- nant peripheral nerve sheath tumors: a study of 10 tumors from nine patients. Cancer Genet Cytogenet. 2006;165:98-105

21. Hoshina D, Abe R, Yoshioka N, et al. Establishment of a novel experimental model of human angiosarcoma and a VEGF-targeting therapeutic experiment. J Dermatol Sci. 2013;70:116-122.

22. Liu G, Yu FX, Kim YC, et al. Kaposi sarcoma-associated herpesvirus promotes tumorigenesis by modulating the Hippo pathway. Oncogene. 2015;34:3536-3546

23. Eisinger-Mathason TS, Mucaj V, Biju KM, et al. Deregulation of the Hippo pathway in soft-tissue sarcoma promotes FOXM1 expression and tumorigenesis. Proc Natl Acad Sci USA. 2015;112:E3402-3411.

24. Gartel AL. Thiostrepton, proteasome inhibitors and FOXM1. Cell Cycle. 2011;10:4341-4342.

25. Gartel AL. Thiazole Antibiotics Siomycin A and Thiostrepton Inhibit the Transcriptional Activity of FOXM1. Front Oncol. 2013;3:150.

26. Li X, Yao R, Yue L, et al. FOXM1 mediates resistance to docetaxel in gastric cancer via up-regulating Stathmin. J Cell Mol Med. 2014;18:811-823. 